\title{
SHORT PERIOD VARIABLES IN THE MAGELLANIC CLOUD CLUSTER NGC 1466
}

\author{
M. V. NORRIS \\ Dunsink Observatory
}

\section{Introduction}

NGC $1466\left(\alpha_{1950}=3^{\mathrm{h}} 44^{\mathrm{m}} \cdot 6, \delta_{1950}=-71^{\circ} 45^{\prime}\right)$ is a globular cluster which appears to be situated between the two Magellanic Clouds. Previous estimates (Gascoigne, 1966) put it at roughly the same distance from us as the LMC, so it is regarded as a member of the Cloud system. It is globular in appearance, and its colour-magnitude diagram confirms this classification. It has a fairly well-developed horizontal branch, and was found by Wesselink (1970) to be quite rich in variables. The metallicity index, $Q$, (van den Bergh, 1967) has a value of -0.36 for NGC 1466 (Andrews and Lloyd Evans, 1971). This would rank it with M5 and NGC 6171 as a cluster of intermediate metal content. This comparison is consistent with the value of $\Delta V$ for the cluster, which, at 2.6 , is representative of the $\Delta V$ values of globular clusters of intermediate metal abundance in the Galaxy.

\section{Observations}

The observational material consists of three sets of plates, taken with different photometric systems:

(i) 21 plates taken in October-December 1969 on the $188-\mathrm{cm}$ telescope of the Radcliffe Observatory. 12 of these were $V$ plates and 9 were $B$ plates.

(ii) 11 plates taken by Wesselink in October-November 1952 on the Radcliffe telescope, using 103a-O emulsion with no filter.

(iii) $4 B$ plates taken by John Graham in November 1969 with the $152-\mathrm{cm}$ telescope at Cerro Tololo.

The three sets of plates were measured on an Askania iris photometer. The faint photoelectric sequence of Gascoigne (1969) was used to perform the reductions. The effects of varying background density were partially eliminated by means of a method outlined by Weaver (1962).

Because of the quasi-systematic way in which colour varies with magnitude in Gascoigne's sequence, it was not possible to use this sequence to determine colour terms for the Radcliffe $B$ and $V$ plates. Instead, Butler's OL sequence in LMC II (Butler, 1971) was used for this purpose. $B$ and $V$ plates of this sequence had been taken with the Radcliffe telescope in 1969 , so that observations of the OL sequence, in the same photometric system as the observations of NGC 1466 were available.

The unfiltered Wesselink plates were reduced by means of a secondary sequence, determined from the reduced measurements of the $B$ and $V$ plates. The colour term, which was quite large and negative, was also derived by means of this secondary 
sequence. The Cerro Tololo plates were measured solely to provide more points on the light curves of the variables. Therefore, only confirmed variables and the stars of Gascoigne's sequence were measured on these plates.

Periods of variables were determined using the $B, V$ and Wesselink sets of plates individually. Periods were adopted only if they agreed between at least two sets of plates; in fact, most of the periods found agreed between the three sets. Periods were found for $9 a b$-type RR Lyrae variables, and one $c$-type was tentatively confirmed. Of these 10 variables, periods had been found for 6 by Wesselink (1971). The periods found for these 6 in the present work agree, to within the limits of accuracy, with Wesselink's values. The period of No. 98, the c-type variable, can be confirmed as 0.353 if it is assumed that the amplitude varies from cycle to cycle.

The number of plates in either colour was not sufficient to construct separate light curves, so it was decided to combine the $B, V$, Wesselink and Cerro Tololo plates to construct one light curve, in $B$, for each variable. This was done by assuming a mean variation of colour with phase, derived from observations of RR Lyrae stars in NGC 6171 by Dickens (1970), and a mean colour derived from rough light curves in $B$ and $V$. In this way, $V$ magnitudes could be converted into $B$ magnitudes at the same phase:

$$
B_{\phi}=V_{\phi}+(B-V)_{\phi}+(\langle B\rangle-\langle V\rangle),
$$

where $(B-V)_{\phi}$ is a smooth normalised function derived from the mean colour curves of the ab-type variables in NGC 6171. For the $c$-type variable in NGC 1466, $(B-V)_{\phi}$ was derived from the $c$-type colour curves in NGC 6171.

The colour derived from the light curves is given by $\langle B\rangle-\langle V\rangle$. This is not the same as the mean of the colour intensity curve, $\langle B-V\rangle$. In fact, on examining Dickens' data, one finds a systematic difference between the two colours, such that $\langle B-V\rangle$ is always redder than $\langle B\rangle-\langle V\rangle$. The mean difference is

$$
\langle B-V\rangle-(\langle B\rangle-\langle V\rangle)=+0.03 .
$$

NGC 1466 is free of any absorption by either Magellanic Cloud, and is subject only to a small amount of Galactic absorption. Extrapolating from the data of Butler (1971) in the wing of the LMC, we assign a reddening of +0 mo2 to the cluster. Thus, the $\langle B\rangle-\langle V\rangle$ values are transformed to the unreddened mean colours, $\langle B-V\rangle_{0}$, by adding $0.03-0.02=0 \%$ m. 01 .

\section{Results}

All the light curves, except that of No. 98 resemble Bailey type- $a b$ R R Lyrae variable light curves, in that they have a sharp rise to maximum light, and a slow descent. No. 109 with the shortest period of the $a b$-types, has a rising branch which is not extremely steep, while No. 116 is an extreme example of an $a$-type variable, with a pronounced dip just before the steep rise to maximum (Figure 1).

The mean period of the $a b$-type variables is 0.533 , indicating that NGC 1466 is a cluster of Oosterhoff Group I (Oosterhoff, 1939). So far, no clusters of Oosterhoff 


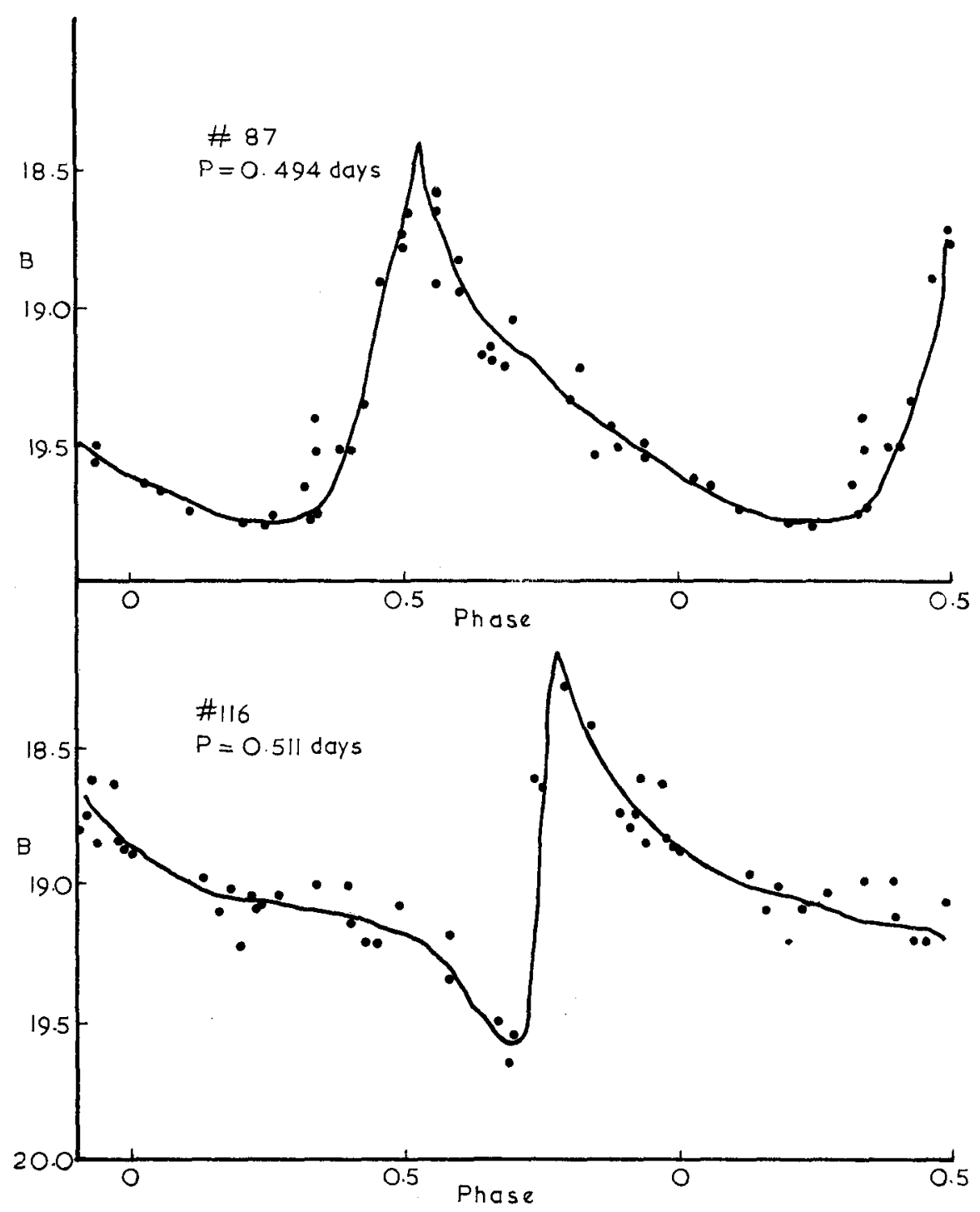

Fig. 1. Light curves of two variables in NGC 1466.

group II, with longer mean $a b$-type periods, have been found in the Magellanic Clouds, suggesting that this system is not as evolved as the Galaxy.

Preston $(1959,1961 \mathrm{a})$ has found that the period-amplitude diagram for a group of RR Lyrae variables depends on the value of the line strength index, $\Delta S$, for the group. The period-amplitude relation for variables in NGC 1466 (Figure 2) lies roughly between those of variables in M5 $(\Delta S=3)$ and variables in M3 $(\Delta S=6)$, i.e. it corresponds roughly to a $\Delta S$-value of 4.5 . Using values of $[M / H]$ found by Preston (1961b) for certain field RR Lyrae variables, and values of $\Delta S$ of the same stars (Preston, 1959), 


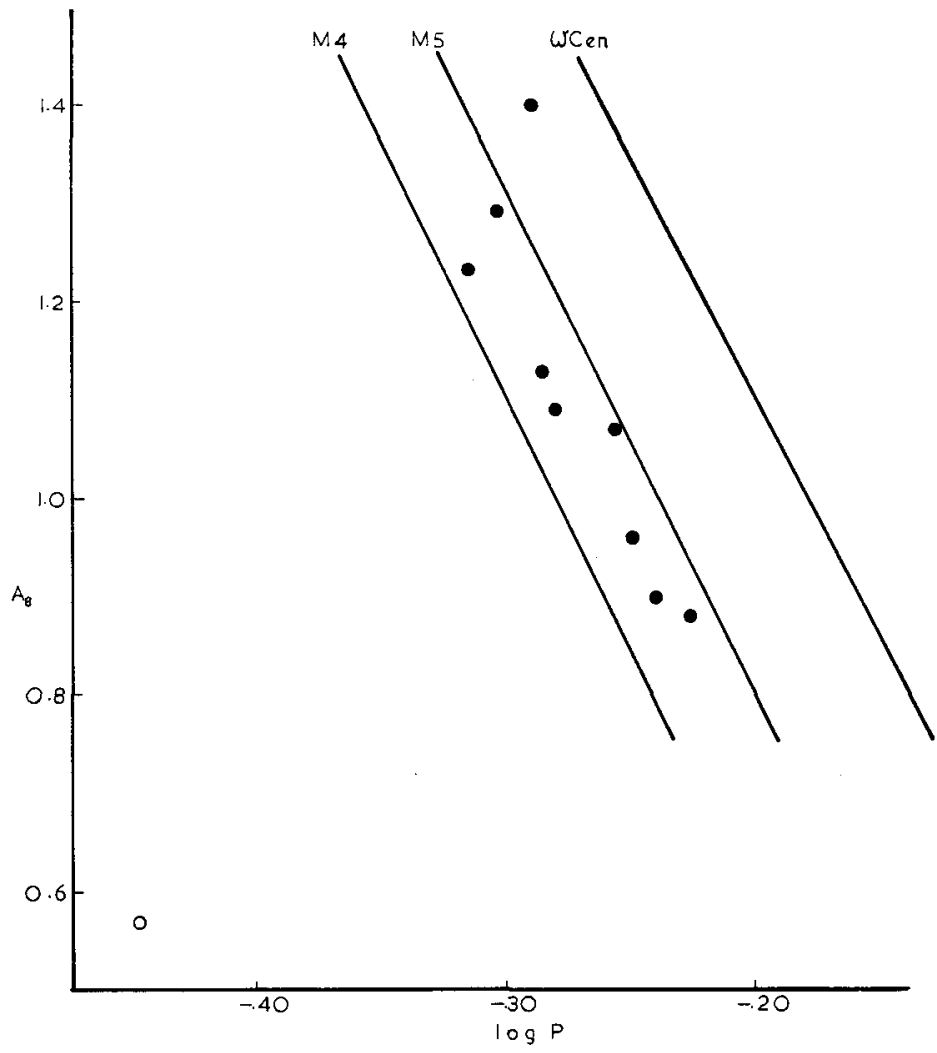

Fig. 2. Period-amplitude diagram of variables in NGC 1466.

these two quantities can be correlated. Using such a relation, a $\Delta S$-value of 4.5 corresponds to a heavy element underabundance by a factor of 6 or 7 in NGC 1466 with respect to the Sun.

The value of $\Delta S$ can be used to obtain a temperature scale appropriate to the amount of line-blanketing in the stars in the NGC 1466. This is done by interpolating in Table II of Dickens (1970) between $\Delta S$-values of 3 and 6, and linearizing the scale in the $\log T_{e}-(B-V)_{0}$ plane. Using this scale, together with the $\log P-(B-V)_{0}$ array for the variables (Figure 3 ) it is possible to derive a mass-luminosity relation from the $P \sqrt{ } \varrho=$ constant analogue of van Albada and Baker (1971):

$$
\log P_{f}=-1.772-0.68 \log M+0.84 \log L+3.48 \log \frac{6500}{T_{e}},
$$

where $M$ and $L$ are in solar units. This procedure yields a relation

$$
\log \frac{M}{L}=-1.89-0.094 M_{\text {bol }} \text {. }
$$




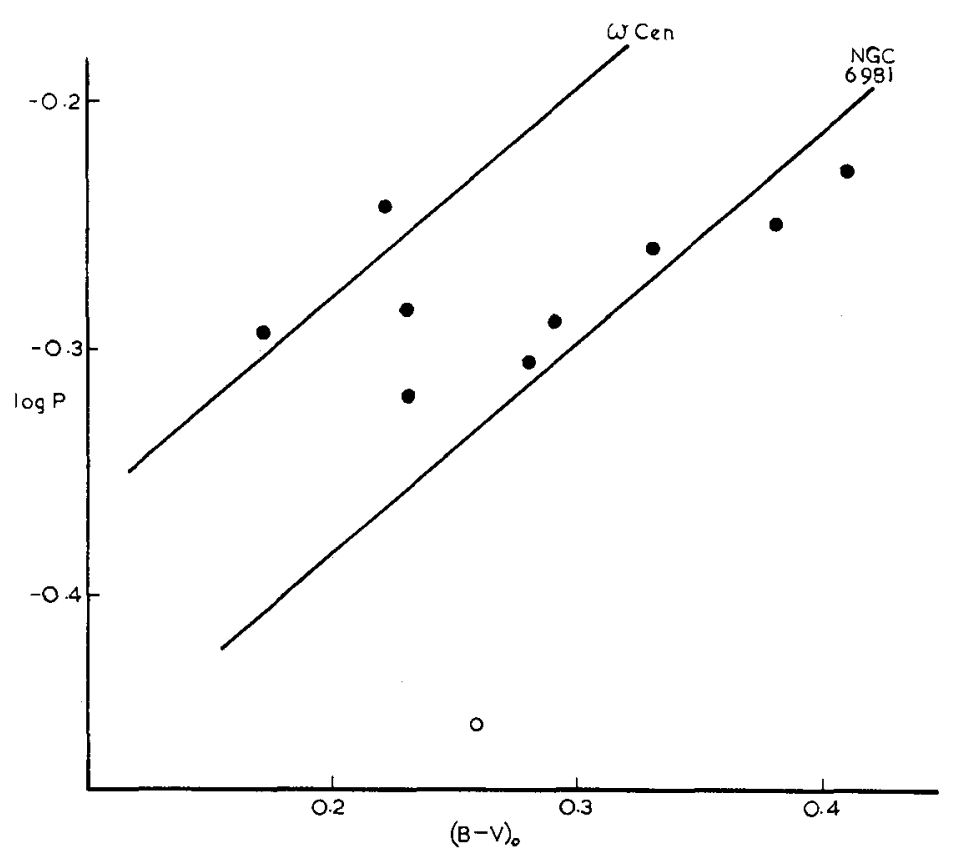

Fig. 3. Period-colour diagram of variables in NGC 1466.

When the value of $M_{\mathrm{bol}}=+0^{\mathrm{m}} \cdot 466$ (see below) is inserted, a mass-luminosity ratio is obtained:

$$
\log \frac{M}{L}=-1.93 \pm 0.09
$$

The absolute magnitude of the variables is derived from Equation (16) of Iben (1971), which is obtained from the results of linear pulsation theory models:

$$
M_{\text {bol }}=\left(1.98 Y-2.5 \log P_{I}-1.07\right) /(0.96-1.1 Y),
$$

where $P_{I}$ is the fundamental period at the intersection of the fundamental and first harmonic 'blue edges' in the luminosity-colour diagram. This is equivalent to the shortest fundamental, or $a b$-type, period in a cluster, which in the case of NGC 1466, is given by

$$
\log P_{I}=-0.320 \text {. }
$$

Inserting this value in Equation (6) gives

$$
M_{\text {bol }}=(1.98 Y-0.28) /(0.96-1.1 Y) .
$$

Equation (8), when combined with Equation (12) (see below), yields the following value for the absolute magnitude of the variables:

$$
M_{\text {bol }}=+0^{\mathrm{m}} \cdot 46 \text {. }
$$


combining this with the mean apparent magnitude of the variables yields an uncorrected distance modulus for the cluster of

$$
\left(V-M_{\text {bol }}\right)=18^{\mathrm{m}} \cdot 70 \pm 0^{\mathrm{m}} \cdot 15
$$

the error being due to the scatter in the apparent magnitudes of the variables. This value for the distance modulus agrees with that found by Gascoigne (1966).

The helium content of the variables is derived from the relation governing the temperature at the blue edge of the instability strip, obtained empirically by Iben (1971) from the results of his linear models:

$$
\begin{aligned}
\log L= & 1.84-5.08 \Delta Y-(12.3-61 \Delta Y) \Delta \log T_{e}+ \\
& +\left[(0.9-0.4 \Delta Y)-(16-33 \Delta Y) \Delta \log T_{e}\right] \log M,
\end{aligned}
$$

where $\Delta Y=Y-0.3$ and $\Delta \log T_{e}=\log T_{e B E}-3.87$.

$T_{e B E}=$ effective temperature at blue edge of instability strip.

Using the temperature scale described above, and the observational evidence that the unreddened mean colour of the bluest variable in the cluster is +0.17 , together with the previously derived mass-luminosity relation (Equation (4)), Equation (11) reduces to

$$
0=-0.110+5.386 \Delta Y+0.351 M_{\text {bol }}+0.116 \Delta Y \cdot M_{\text {bol }} .
$$

When combined with Equation (8), this yields

$$
Y=0.29 \text {, }
$$

i.e., a normal Helium content for a Population II object in the Magellanic Clouds.

\section{Acknowledgements}

The author is indebted to Prof. P. A. Wayman for his encouragement and invaluable help during the present work. Thanks are also due to the Dublin Institute for Advanced Studies for their financial support.

\section{References}

Albada, J. D. van and Baker, N. H.: 1971, Astrophys. J. 169, 311.

Andrews, P. J. and Lloyd Evans, T.: 1971 in A. B. Muller (ed.), The Magellanic Clouds, Reidel Co., Dordrecht, p. 88.

Bergh, S. van den: 1967, Astron. J. 72, 70.

Butler, C. J.: 1971, Thesis, Trinity College, Dublin.

Dickens, R. J.: 1970, Astrophys. J. Suppl. 22, 249.

Gascoigne, S. C. B.: 1966, Monthly Notices Roy. Astron. Soc. 134, 59.

Gascoigne, S. C. B.: 1971, Private communication.

Iben, I.: 1971, Publ. Astron. Soc. Pacific 83, 697.

Oosterhoff, P. Th.: 1939, Observatory 62, 104.

Preston, G. W.: 1959, Astrophys. J. 130, 507.

Preston, G. W.: 1961a, Astrophys. J. 134, 651.

Preston, G. W.: 1961b, Astrophys, J. 134, 633.

Weaver, H.: 1962, Handbuch der Physik 54, 130.

Wesselink, A. J.: 1970, Private communication.

Wesselink, A. J.: 1971, Monthly Notices Roy. Astron. Soc. 152, 159. 


\section{DISCUSSION}

Graham: (1) Is not NGC 1466 slightly nearer to the LMC than the SMC? (2) Did you find any faint RR Lyraes in the general field outside the cluster?

Norris: In terms of angular separation, NGC 1466 is closer to the Large than to the Small Cloud. This, combined with the fact that the cluster appears to be at the same distance from us as the LMC, would place NGC 1466 linearly closer to the LMC than to the SMC. (2) The Radcliffe plates have been blinked almost to the edges, i.e., to about $70^{\prime}$ from the centre of NGC 1466. No new variables were found in this region, which at the distance of the cluster, has a radius of about $300 \mathrm{pc}$. 